\title{
Praxis - Anwendungen - Lösungen
}

Das Schutzprofil für digitale Videoanlagen

\section{Sicherheits- und Datenschutz-Management}

Transparenz der Risiken beim Einsatz von IT

\section{Grundlagen - Technik und Methoden}

Eine für alles:

Unified-Architekturen vereinfachen Archivierung und Compliance

Mehr Sicherheit durch Windows Vista? (IV):

Der Internet Explorer 7

Herausgeberbeirat

Wilfried Reiners, PRW Rechtsanwälte

Sebastian Schreiber, SySS GmbH

Ulrich Emmert, esb Rechtsanwälte

Andreas Bichlmeir, Ingram Micro GmbH

Stefan Gehrke, Mcert Deutsche

Gesellschaft für IT-Sicherheit mbH

Rainer Hilsenbeck, FocusIT

Holger Prescher, MainSkill

Technologies $\mathrm{GmbH}$ \& Co. KG

Tobias R. Hoffmann, Aviconet $\mathrm{GmbH}$

Redaktion

Chefredaktion:

Wolfgang Dumke, V.i.S.d.P

Ltd. Redakteur:

Thomas Böcker
Dr. Christoph Busch, CAST e. V. Harald Summa, eco Verband der Deutschen Internetwirtschaft e.V.

Isabel Münch, BSI - Bundesamt für Sicherheit in der Informationstechnik Boris Bärmichl, KoSiB eG,

Kompetenzzentrum für Sicherheit Matthias Jänichen, perComp-Verlag $\mathrm{GmbH}$

Grafik \& Layout

Druckerei Klein + Hiese GmbH

Wilhelmstraße 22

63911 Klingenberg

\section{Online-Service} www.it-sd.com 